\title{
Design and Development of Multimedia Teaching System based on C/S Mode
}

\author{
Meilian Chen ${ }^{1}$ Yan Ma ${ }^{1 *}$ \\ 1.College of Computer and Information Science, Chongqing Normal University, Chongqing \\ *Email: cqnu_mayan@163.com
}

\begin{abstract}
:
Based on the existing campus network this paper proposes the scheme established a Web-based multimedia teaching network platform, introduces the design idea and overall structure of the system, and analyzes the function and composition of each module. It introduces the functions as a multimedia teaching platform and finally analyzes the key technologies of the multimedia platform based on campus network: Web technologies of Client/Server structure and Browse/Server structure.
\end{abstract}

Keywords: Remote network; C/S mode; B/S mode; Web Technology

\section{Introduction}

The rise of computer-centric Internet and multimedia technology not only has brought great impact to modern education, but also has injected new life into it to produce a major leap forward. The field of education will be one of the most valuable and the most promising applications areas of Internet application. With network technology and multimedia technology, distance education represents the direction, trends and the future of educational technology.

Currently the implementation of distance education on the Internet is generally based on WWW (World Wide Web) technology[1]. With the development of network and multimedia technology, various education resources and informations on the Internet enrich constantly, students and teachers who participate in distance education are also more and more, the effect of distance education support platform is becoming more and more important. Distance education support platform is a system which manages and uses a variety of educational resources on the network by Web technology and manages teachers and students who participate in distance education. It includes a variety of hardware resources, servers working platforms, Web servers, database servers, and storage organizations of interface technology of Web servers and database servers and a variety of resources and informations, etc[2].

\section{The main technical characteristics of distance education platform}

The system structure of distance education supporting platform includes two modes. One is the traditional mode based on Client / Server (C / S), which mainly solves system maintenance. Another is the new mode based on Browser / Server (B / S), which mainly solves issue of various resources and informations on the Web, queries, and remote call[3]. Among them B/S model is for the majority of users, so it is the key problem to be resolved. The B / S model is based on "thin" client, an intermediate layer which contains application logic and three-layer structure of a database layer, that is, from the client browser, Web server and database server consists of three parts as shown in Figure 1 shown.

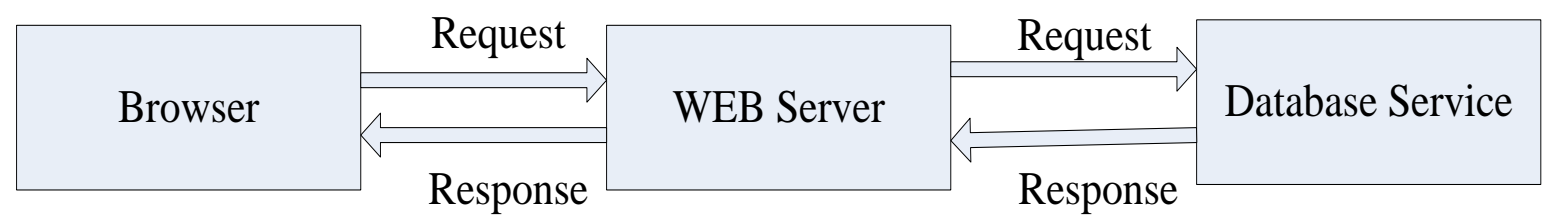

Figure 1: platform structure of distance education 
Its main characteristics are as follows:

(1) The system is easy to extend and maintain. The B/S structure has great flexibility, which not only can combine the Web server and database server but also can connect a plurality of database servers at the same time..

(2) Realizing the cross-platform system integration service truly. Through unified protocols and standards, a variety of network operating systems (such as UNIX, NetWare, Windows NT, Linux, etc.) and multiple database management systems (SQL Server, Sybase, Oracle, Informix, etc.) fully integrate in a B/S structure, so as to provide users with a clear and unified message service interface.

(3) Greatly reducing the demands for network bandwidth. Due to the adoption of B/S structure, the client just do present and input of data, business logic is fully implemented on the server, thereby significantly reducing the network load.

\section{The overall structure of the system}

To meet the needs of modern distance education and online learning, we built a experimental system based on $\mathrm{B} / \mathrm{S}$ structure and the Web as a platform. It is an integrated organic system which contains hardware, software, teaching content, teaching management organization. Based on the campus network as the basis this system establishes the Web teaching sites, teaching content is presented in the form of Web courseware on pages to achieve teaching[4].

The system consists of the following modules: access module, switching module, server module, network management and billing module, courseware and teaching module, teaching data resources module, etc, and its structure diagram is as shown in Figure 2:

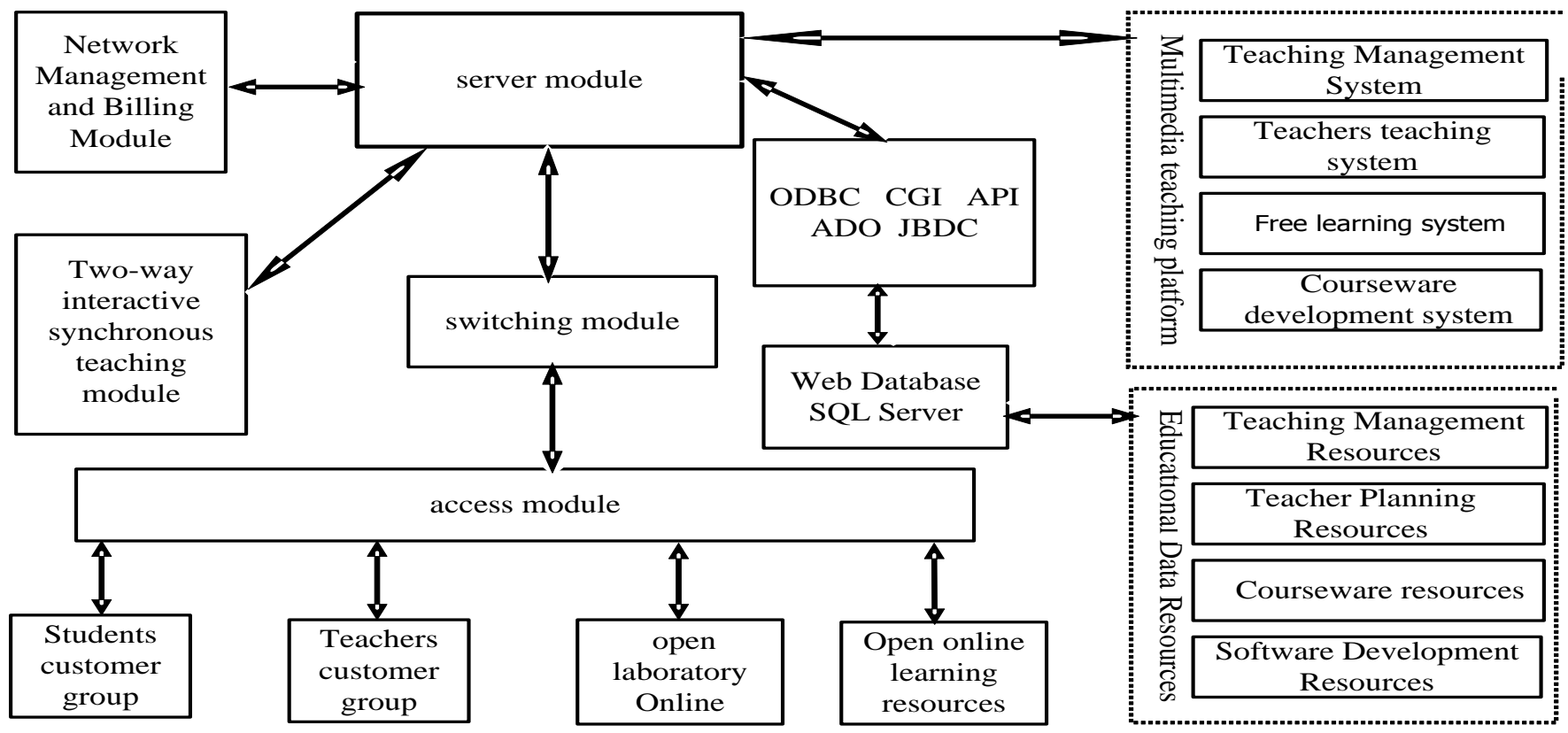

Figure 2: Teaching network system composition principle based on B/S

In this system, the role and function of each main module are as follows:

Access module: Its main function is to enable students and teachers to access the network resources by a variety of ways, so as to achieve the purpose of teaching or learning, its main device is routers and access servers. The main function of the router is connecting the client's local area network with campus network through a special line. The main role of access server is to make the user access online educational resources by dialing way, so as to achieve the purpose of online learning.

Switching modules: It is the core of the entire network connection and the transmission. the main equipment has a backbone switch, branch hub, network cable and optical fiber connected to each module, the backbone network is composed by them. 
Server module: It is mainly responsible for the collection, storage, release of information, they are the main entities providing teaching and information service for the user, generally including:

WEB server: Using the WWW service function above IIS4.0 and MS SQL Server as the data directory database, accessing and managing teaching data resources through the IDC mode.

FTP server: Using the FTP service function above IIS4.0, managing and publishing various courseware online in a hierarchical manner.

E-mail server: Through email management, setting up email address for teachers and students to carry out teaching feedback effects.

DNS server, database server, and so on: They can be borne by one or more high performance computer.

VOD server: Providing the video output requested by network users, requiring that the server should have a huge external storage, generally can use RAID way or other. Management software can be MediaBase, it uses RSVP protocol, under the Informix database, and it is suitable for ATM and fast ethernet.

Network management and billing module: Network management module is mainly to monitor the entire network, performance monitoring, fault warning and diagnosis, etc. The billing module is mainly used to record the tariff information of Internet users to control the compensation for the use of network resources.

Courseware and teaching module: the main role is to develop and maintain teaching content and educational resources online, and organizing teaching according to the resources. The teaching module with two-way interactive synchronous teaching function is a high-speed data network two-way video conversation system, the core equipment of this module is video conference terminals based on the related standard (such as h.323), MCU (multipoint control unit), camera, etc[5]. MCU is a multiport equipment, it can make a plurality of video terminal to communicate with each other in one session, its main control functions including audio mixing, audio switching, video mixing and video switching. By it teacher's explanation can be transferred to the remote multimedia classroom real-timely, teacher in the lecture can see the listening effects of the students in the remote classroom and receive their inquiries, as for classroom teaching in the local[6].

In this system, the server uses Windows NT4.0 Server, Windows NT Server Pack 3, IIS 4, SQL Server 7 , Active Server Pages.

\section{The key technology}

\subsection{The establishment of the cognitive model}

ICAI emphasizes that according to students' different cognitive model preparing different teaching contents for them, it is used to realize the individualized teaching and heuristic teaching. Restricted by the cognitive psychology and pedagogy development, it is unable to accurately establish a single person's cognitive model.

According to the student's cognitive ability, cognitive model is divided into high, medium and low three categories. Lesson plans of the same content are also divided into high, medium and low three difficulty and respectively provided to the students who have appropriate cognitive abilities. After learning the teaching content of each section, the system will ask student a number of related questions and determines his new cognitive model according to the accuracy.

\subsection{The realization of intelligent teaching process}

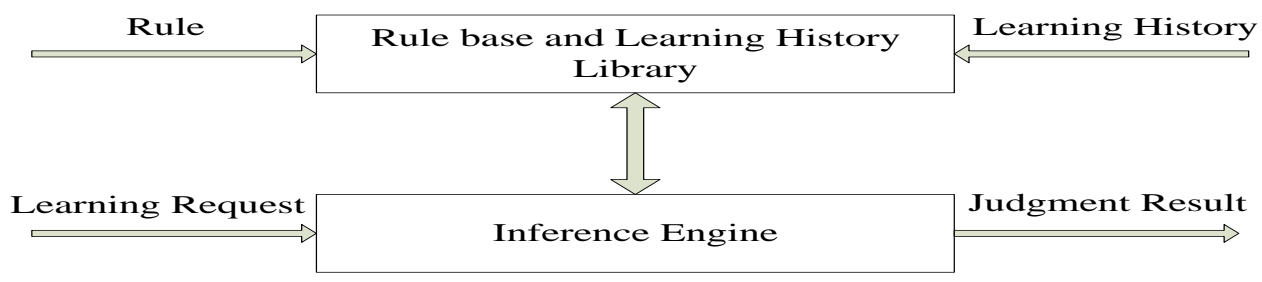

Figure 3: ICAI intelligence implementations 
With reference to the design method of expert system, implementing the intelligent functions of ICAI courseware system. In this system, organizing a corresponding rule library , fact library (Learning History Library) and reasoning mechanisms to achieve, as shown in Figure 3. A brief introduction to the reasoning mechanism is shown below. Rule library from the books, chapters, sections, hypertext lesson plans address, and a number of prerequisite threshold composition, which reflects section to learn preconditions to be met. Studying history library consists of books, chapters, sections, cognitive level, the number of components have learned, which reflects the students' cognitive abilities. Rule library is modified by the teacher. The students' learning history is organized by a table for each student, after learning its contents, the client software would modify the basis of the student's mastery of knowledge points automatically by the students. According to the rules provided by the library rules and history library provided students the facts, inference module dedicated to make judgments. After teaching learning content in each section, based on the accuracy of exercise answers, we will divide their cognitive abilities into high, medium and low three-level, and the result is stored in student learning history library. According to their cognitive abilities, the system will be different as he prepares the corresponding difficulty of teaching content when the students learn to use the system the next time. It is obvious that such a measure of cognitive ability is dynamic. Students who do not want the progress of science by the default system, then ask students to choose learning content. If the selected content has been learned, it was obtained for his learning content with their level of preparation; otherwise it will depend on the rule library and learning history content to determine if it can be learned.

\subsection{Given threshold}

When students complete contents of a section, the score is less than the section has the threshold, the system does not have learned the festival logo. So the student logs the next time, the system will force him to re-learn the contents of the section. Typically, the section has a special importance in the chapter has a higher threshold in this discipline. The following is a important method of the course evaluation:

$$
\operatorname{Important}(\mathrm{N})=\mathrm{IN}(\mathrm{N})+\mathrm{OUT}(\mathrm{N})
$$

Important(N)—Status of the section $\mathrm{N}$ in this course(The book is in the arrangement of water situation in number);

$\mathrm{IN}(\mathrm{N})$ - In this course, the number of the knowledge which has effect of section $\mathrm{N}$;

OUT(N)_- In this course, the knowledge of section $\mathrm{N}$ affecting the rest of the number of the section.

When in the course of all $\operatorname{Important}(\mathrm{N})$ calculation is completed, then gives a section of the threshold.

Threshold Valve(N) represents the situation in hundreds of copies, less than 1 and Proportional with $\operatorname{Important}(\mathrm{N})$.

Let Right (N) indicates that a student who had learned section $\mathrm{N}$, and the accuracy of the system after answering the questions. Variable Ruler=1-Valve(N). A cognitive model modification rules are as follows:

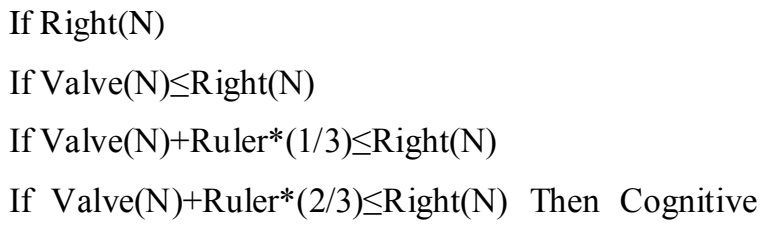
ability as "high"

\section{Teaching evaluation}

Intelligence Computer Assisted Instruction-ICAI has some of the following features[7]:

(1) Generate a variety of problems and exercises automatically

(2)According to the students' learning levels and the learning to choose and adjust content and schedule;

(3) Based on the understanding of teaching content, solve problems, generate answers automatically;

(4) Has the ability to produce and understand natural language, in order to achieve teaching Answering System more freedom, initiative to improve the human-computer interaction; 
(5) To explain the teaching content advisory capacity ;

(6) Diagnosis of students' mistakes, analyze the causes and take corrective measures ;

(7) To evaluate students learning behavior ;

(8) To evaluate the teachers' behavior。

All of the above features with ICAI system is perfect, but it is difficult to achieve. Generally believed that the system has one or several characteristic above, it can be called the ICAI.

We use the following method to achieve the level of students' individual learning evaluation。

$\mathrm{n}$ : The total number of users of the system ;

$\mathrm{Xi}$ : The test scores of i student.

define :

$\bar{X}=\frac{\sum_{i=1}^{n} X_{i}}{n} \quad S=\sqrt{\frac{\sum_{i=1}^{n}\left(X_{i}-\bar{X}\right)^{2}}{n}} \quad Z_{i}=\frac{X_{i}-\bar{X}}{S}$

By definition, $S$ is the standard deviation of the overall student achievement. $\mathrm{Zi}$ reflects the $\mathrm{i}$ student achievement and the average difference is several times of the standard deviation. With $\mathrm{Zi}$ to measure the difficulty of exam papers will not be affected by the impact of values, more persuasive than $\mathrm{Xi}$. Because standard scores ( $\mathrm{Zi})$ is a reference zero (average) position is fixed, per unit length (standard deviation) the same statistics [8]. Different times of the original test scores converted into standard scores, you can do compared with each other. If a student test scores twice raw scores were:80,90, standard scores were:2.5, 2. It could not concluded that the student learning progress by the second raw score higher than the first. Assertion is correct: Because the second test standard score lower than the first, the student academic performance regressed. Experience shows that, the numerical standard score between $-3 \sim+3$, When the standard score is positive, it means that their level of learning level value; contrary, it indicates the level of learning under the level value.

This work is sponsored by Chongqing Education Commission Research Project (NO:KJ120617,KJ120634), Chongqing Graduate Education Reform Project (Yjg123039), and Chongqing Normal University Teaching Reform Research Project in Higher Education (NO:201334).

\section{- References}

[1] Jiandong Wang. "Analysis of multimedia courseware development technology and method based on WWW." [J], Modern agriculture,2010(4)

[2] Zhurong Zhou, Xiaozhen Zhang. "The Comprehensive Integration of ICAI and Hypertext." Journal of Computer Research and Development, Vol.35,No.8 Aug.1998

[3] Xiangyong Guo. "The Campus Multimedia Teaching Network Database Design, Construction and Application Research.” Application Research of Computers, 2001.1, 97-100

[4] J uanjuan Zhang, Weixin Wu. "Design and Development of Multimedia Teaching System based on WEB.” Fujian computer, 2009 (4) , 130 131

[5] Shengjian Liu, Zhong Zheng. "The Application of Web Technologies to Build Multimedia Teaching Platform online." Computer Science, 2000,27 (9) ,371-373

[6] Yin Chen, Qinming He, Shenkang Wang. "The Intelligent Computer Assisted Instruction System based on the Structure of Hypertext." Journal of Computer Research and Development, Vol.35,No.5 May.1998

[7] L an Zhang, Zhengyan Lu. "Development and application of multimedia courseware in the teaching of Professional skills." Science and technology innovation herald, 2012(17)

[8] Stephen Wynkoop, Special Edition Using Microsoft SQL Server 6.5 Second Edition, Copyright 1997 By Que Corporati 